\title{
Mindful attention awareness and cognitive defusion are indirectly associated with less PTSD-like symptoms via reduced maladaptive posttraumatic cognitions and avoidance coping
}

\author{
Daisuke Ito ${ }^{1}$ (D) Asuka Watanabe ${ }^{2}$ (D) Kaori Osawa ${ }^{3}$ (D)
}

Accepted: 19 February 2021 / Published online: 25 February 2021

(C) The Author(s) 2021

\begin{abstract}
Recently, the effects of mindfulness-based interventions for posttraumatic stress disorder (PTSD) have been studied. However, we lack knowledge of the processes or mechanisms through which mindfulness influences resilience or promotes recovery following exposure to trauma. In this study, we examine the process by which mindfulness and cognitive-behavioral factors such as posttraumatic negative cognition and avoidance behaviors are associated with reduced PTSD-like symptoms. A questionnaire survey was conducted with 885 university students, using the Trauma Experience Questionnaire, Mindful Attention Awareness Scale, Cognitive Fusion Questionnaire, Post-Traumatic Stress Disorder Checklist, revised Ways of Coping Checklist, and a brief version of the Posttraumatic Cognitions Inventory. Data from 254 participants who experienced trauma with PTSDlike symptoms were analyzed. The final model using SEM indicated no direct association of mindful attention awareness on PTSD-like symptoms, a small direct association of cognitive defusion on PTSD-like symptoms, and a large indirect association mediated by posttraumatic negative cognitions and avoidance coping. Owing to the study's cross-sectional design, strong conclusions cannot be drawn regarding the causal relationship of mindfulness and cognitive-behavioral factors or generalizations. However, our findings suggest that it would be worthwhile to continue to clarify the mechanism of mindfulness and to investigate its use in the treatment of PTSD. In the future, a highly accurate study design will hopefully be used to examine the mechanisms of several different factors associated with improvement in posttraumatic stress symptoms, preferably including them in the same model.
\end{abstract}

Keywords Mindfulness skills $\cdot$ Cognitive-behavioral factors $\cdot$ PTSD-like symptoms $\cdot$ Structural equation modelling

Daisuke Ito

dito@hyogo-u.ac.jp

Asuka Watanabe

d20208h@hyogo-u.ac.jp

Kaori Osawa

caosawa@konan-u.ac.jp

1 Department Clinical Psychology, Graduate School of Education, Hyogo University of Teacher Education, 1-5-7

Higashikawasaki-cho, Kobe, Hyogo, Japan

2 Department of School Counseling and Human Developmental Support, The Joint Graduate School in Science of School Education, Hyogo University of Teacher Education, 1-5-7 Higashikawasaki-cho, Kobe, Hyogo, Japan

3 Department of Human Sciences, Faculty of Letters, Konan University, 8-9-1 Okamoto, Kobe, Hyogo, Japan
Posttraumatic stress disorder (PTSD) is a chronic, often debilitating, mental health condition that may develop after a traumatic event, such as a natural disaster, traffic accident, sexual assault, or unexpected loss of a loved one. The past year and lifetime prevalence rates of PTSD in the US community samples have been reported to be $4.7 \%$ and $8.3 \%$, respectively (Kilpatrick et al., 2013). PTSD is associated with physical health issues, social dysfunction, and work difficulties. However, most people who experience trauma with posttraumatic stress symptoms (PTSS) do not need treatment and recover spontaneously. Research on symptom trajectories indicates that many who develop PTSD experience some level of recovery (Reijnen, Rademaker, Vermetten, \& Geuze, 2015). Thus, not all people who experience acute distress or meet the diagnostic criteria for PTSD at one time will continue to meet diagnostic criteria over time.

Since the introduction of PTSD into the psychiatric nosology in 1980, considerable debate has arisen over whether PTSS can, 
in fact, be clearly discriminated as pathological (i.e., PTSD) versus normative. Recent taxometric research supports the concept of PTSD as a graded dimensional, non-categorical model (Kliem et al., 2016) that is positioned on a continuous spectrum of PTSS. Therefore, it is useful to include individuals in research and clinical services, even if their symptoms do not meet diagnostic criteria. For example, any dichotomization (e.g., dividing individuals into pathological vs. nonpathological categories) of the PTSD construct in research will create an unnatural break in the data, thereby limiting construct validity, and leading to an unnecessary loss of information and statistical power (BromanFulks et al., 2009). Individuals and organizations should also be wary of using PTSD diagnostic criteria to decide the eligibility for receiving psychological services. In fact, given that many individuals who experience PTSS, but do not meet the full diagnostic criteria for PTSD, report significant impairment in occupational and psychosocial functioning (Marshall et al., 2001). Hence, public health and economic significance of dealing with sub-diagnostic PTSS in research and clinical practice is high.

Psychological research has attempted to clarify the psychosocial risk factors related to the maintenance of PTSS and apply them to treatment. Notably, Xue et al. (2015) suggested that psychosocial risk factors experienced during or after trauma (e.g., lack of social support, peri-traumatic dissociation) were more strongly associated with PTSD than sociodemographic factors (e.g., gender, age), pointing to the importance of potentially malleable psychosocial factors.

In the cognitive-behavioral model, trauma-related cognitive and behavioral factors strongly predict the persistence of PTSS (Ehlers \& Clark, 2000). Currently, this model is one of the most useful psychological models (Ehlers \& Clark, 2000). These models suggest that PTSS are maintained when individuals process trauma in a way that leads to a sense of serious, current threat, which arises because of excessively negative cognition of trauma and/or its sequelae. Moreover, changes in negative cognition are prevented by problematic behavioral and cognitive strategies, such as avoidance coping. Therefore, traumatic cognition that maintains a sense of threat and exacerbates PTSS and avoidance coping that prevents reassessment of traumatic cognition should be modified. Several techniques used in trauma-related cognitive-behavioral interventions can facilitate changes in these areas (e.g., Foa \& Rothbaum, 1998; Zalta et al., 2014).

In fact, the American Psychiatric Association (APA, 2013) guidelines strongly recommend interventions that directly address traumatic cognition and avoidance behaviors related to a traumatic event, such as Trauma-focused Cognitive Behavioral Therapy (TF-CBT), Prolonged Exposure (PE), and Cognitive Processing Therapy (CPT). Although PE and CPT have been associated with clinically significant symptom relief and shown to have large effect sizes (Steenkamp, Litz, Hoge, \& Marmar, 2015), a substantial proportion of patients continue to experience considerable residual symptoms or retain their PTSD diagnosis following treatment (Bradley, Greene, Russ, Dutra, \& Westen, 2005). For example, recent studies report that attrition rates for leading treatment interventions fall between $35 \%$ and $40 \%$ (Goetter et al., 2015). Additionally, although dropout rates do not differ between PE and other psychotherapies (Hembree et al., 2003) and safe interventions, some resistance to interventions incorporating exposure to trauma-related stimuli has been noted among some mental health clinicians, for example, concerns about potential PTSS exacerbation (Zayfert et al., 2005). Patients also express resistance to exposure to trauma-related stimuli, including concerns about the loss of a sense of control (Becker, Darius, \& Schaumberg, 2007). Thus, although traumafocused treatments are considered effective for PTSD, the need for potential adjunctive or alternative treatments is evident.

Recently, attention has been focused on mindfulness-based training as an alternative or supplement to CBT. Mindfulness is commonly defined as focusing on present-moment experiences with a mental stance of receptivity and acceptance (Kabat-Zinn, 1994). One of the most common form of mindfulness training is mindfulness-based stress reduction (MBSR), a structured group program of mindfulness training developed by KabatZinn (1990). Meta-analyses have shown that MBSR improves the health status of a wide spectrum of clinical populations (e.g., depression, anxiety, pain, heart disease, and cancer) and stressed nonclinical groups (Grossman, Nieman, Schmidt, \& Walach, 2004). PTSD is no exception; some studies suggest that training aimed at improving one's degree of mindfulness is as effective for PTSD as conventional CBT (Boyd, Lanius, \& McKinnon, 2018). In addition to being explored as an intervention, mindfulness skills are negatively correlated with PTSS (Boden et al., 2012) and serve as a protective factor against developing trauma-related psychopathology (Nitzan-Assayag, Aderka, \& Bernstein, 2015). Pre-traumatic mindful tendencies promote awareness and acceptance of posttraumatic responses, thereby possibly reducing symptoms such as re-experiencing, hyperarousal reactions, and avoidance of trauma-related stimu$\mathrm{li}$, as well as preventing the onset and maintenance of PTSD (Thompson, Arnkoff, \& Glass, 2011). These studies suggest that increasing one's degree of mindfulness has a preventive or therapeutic effect for individuals with PTSS and may improve mental health and social functioning.

There are some theoretical explanations for how mindfulness improves PTSS, although there are fewer studies from a clinical psychological viewpoint than neuropsychological studies (Boyd et al., 2018). For example, Shapiro, Carlson, Astin, and Freedman (2006) suggest that the three axioms, IAA, are the fundamental components of mindfulness. Intentionally (I) attending (A) with openness and nonjudgmentalness (A) leads to a significant shift in perspective, which they have termed as reperceiving, and results in a positive effect. One of the fundamental components of mindfulness is mindful attention awareness as defined by 
Brown and Ryan (2003) who developed the Mindful Attention Awareness Scale (MAAS). Attention is the focus of awareness on a particular stimulus, and it can increase sensitivity to that stimulus (Westen, 1999). Awareness is then accompanied by awareness of extrinsic and intrinsic stimuli to the body and mind, and it is a direct bridge between us and the events and experiences around us (Brown, Ryan, \& Creswell, 2007). Furthermore, by having mindful attention awareness with extrinsic and intrinsic stimuli such as thoughts and feelings. it is also important to understand that what we think about is not necessarily reality itself. In mindfulness, distancing from thoughts is known as "cognitive defusion" and its counter process is "cognitive fusion," which is the tendency for behavior to be overly regulated and influenced by cognition (Gillanders et al., 2014). When "cognitive fused," a person acts on thoughts as though they are literally true; cognitive events dominate behavior and experience over other sources of behavioral regulation; and as a result, a variety of distress is easily caused. For example, trauma-related stimuli can easily produce intrusive thoughts in PTSD, which are exacerbated PTSS caused by the confusion of the content of those thoughts as real or happening in reality, without being aware that they are simply thoughts. Mindfulness-driven cognitive defusion driven may facilitate the ability to view memories related to traumatic experiences as transient memories rather than currently threatening events and less automatic maladaptive responding to trauma cues (Nitzan-Assayag et al., 2015). Thus, mindfulness-based interventions intend to increase mindful attention awareness and reduce cognitive fusion to enable people to recognize unpleasant emotions or memories in a non-judgmental manner (Bishop et al., 2004).

Such techniques based on mindfulness are a different approach to traditional CBT, which aims to directly alter negative cognition and maladaptive behavior that contributes to the maintenance of PTSS (e.g., Hembree \& Foa, 2000). However, as mentioned earlier, drawing mindful attention to the present moment allows one to escape traumatic memories and cognitive fusion between painful thinking and real-life events or experiences, consequently engaging with them in different ways (Sears \& Chard, 2016). For example, mindfulness may reduce ruminative tendencies, thus reducing anxious arousal and anhedonia; and a non-judgmental outlook may promote willingness to approach fear-provoking stimuli, leading to reduced avoidance (Lang et al., 2012). Mindfulness may also promote awareness of a variety of stimuli and update information, which may result in the modification of posttraumatic negative cognition (e.g., negative beliefs about the world and self, self-blame) and improve PTSS (Nitzan-Assayag et al., 2015). This overview on the mechanism of effect of mindfulness on PTSD demonstrates that having mindfulness skills, such as mindful attention awareness and cognitive defusion, may reduce avoidance behaviors and negative cognitions, which contribute significantly to the maintenance of PTSD.
Despite this promising theory, we lack knowledge of the processes or mechanisms through which mindfulness influences resilience or promotes recovery following exposure to trauma. In particular, to the best of our knowledge, there are no empirical studies that examine how are mindfulness and cognitive-behavioral factors such as posttraumatic negative cognition and avoidance behaviors associated with reduced PTSS. However, a recent systematic review (Kangaslampi \& Peltonen, 2019) suggests that improvement in maladaptive trauma-related cognitions is a core factor in recovery from PTSS. It may be influenced in part by reduced avoidance behaviors due to exposure to traumatic stimuli and reduced sensitivity to threatening stimuli due to non-trauma-focused interventions such as mindfulness and would be useful to consider. Thus, it will help clarify the role of each mechanism to study several potential mechanisms (e.g., mindfulness and/or cognitive-behavioral factors) in analyses and preferably include them in the same models (Hayes \& Rockwood, 2016).

Therefore, we conducted a questionnaire survey of trauma survivors and used structural equation modeling (SEM) to examine a theoretical model, based on previous research, which suggests that mindfulness and cognitive-behavioral factors are associated with reduced PTSS. The A summary of the theoretical model is presented in Fig. 1. The theoretical models consists of (1) direct negative associations of mindful attention awareness on PTSS, and direct positive associations of cognitive fusion on PTSS (e.g., Boden et al., 2012; Nitzan-Assayag et al., 2015) and (2) indirect associations of mindful attention awareness and cognitive fusion on PTSS through mediation of cognitive-behavioral factors, such as avoidance coping and posttraumatic cognition, based on previous research on mindfulness (e.g., Bernstein et al., 2015; Lang et al., 2012; Sears \& Chard, 2016). Additionally, we predicted that (3) avoidance coping positively affects posttraumatic cognition based on maladaptive behavioral coping, preventing changes in negative cognition of trauma (Ehlers \& Clark, 2000), and (4) direct positive associations of avoidance coping and traumatic cognition on PTSS, based on previous studies of CBT for PTSD (e.g., Foa \& Rothbaum, 1998; Zalta et al., 2014). In general, SEM is used as a confirmatory rather than an exploratory analysis. However, the associations of mindfulness tendencies mediated by cognitive-behavioral factors on PTSS have not been fully tested in previous studies, and the hypothesis model may need to be modified. Therefore, in this study, after testing the model fit shown in Fig. 1, we conducted an exploratory examination of a more valid model with reference to the background theory.

As already mentioned, the concept of PTSD has shown the validity of interpreting it as a graded dimensional model (Kliem et al., 2016) that is positioned on a continuous spectrum of PTSS, and therefore is useful even in studies of community samples rather than clinical groups of PTSD. In other words, although we investigated undergraduate and graduate students who had experienced traumatic events, the findings 


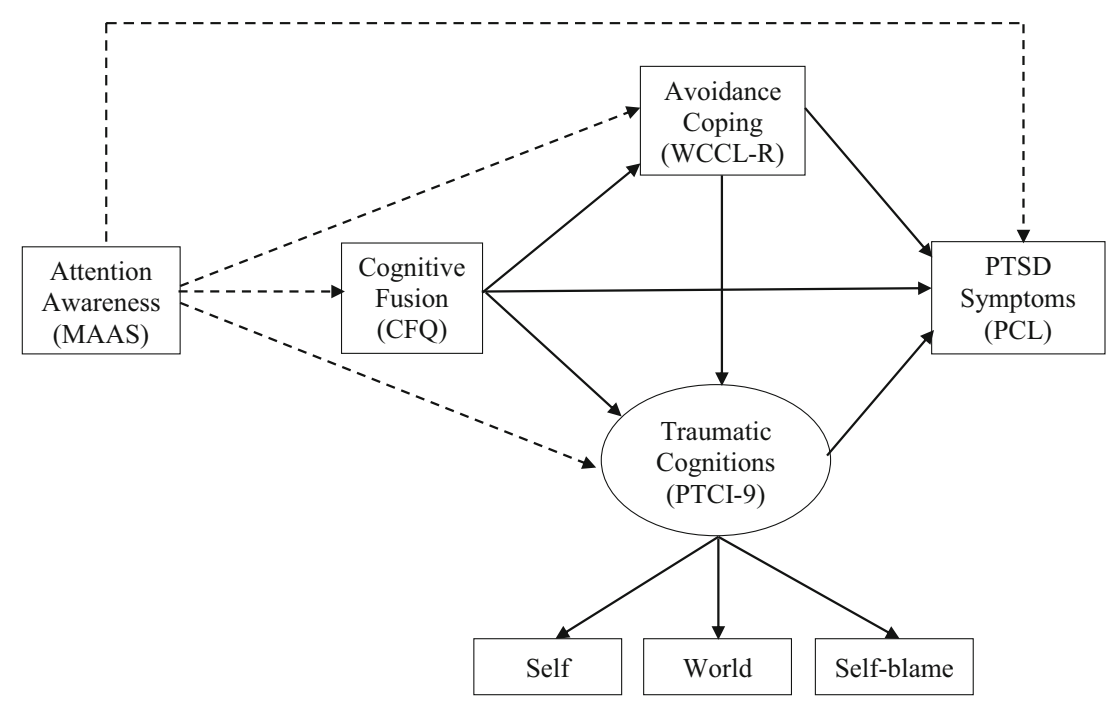

Fig. 1 The Expected Model on the Association of Mindful Attention Awareness and Cognitive Fusion Associated with PTSD-like Symptoms via Avoidance Coping and Posttraumatic Cognitions among Japanese with Traumatic Experiences. Note. Dotted lines represent negative effect pathway and bold lines represent positive effect pathway. MAAS = Mindful Attention Awareness Scale, CFQ = Cognitive Fusion Questionnaire, WCCL-R = Ways of Coping Checklist-Revised, PTCI-9 = Brief Version of the Posttraumatic Cognitions Inventory, $\mathrm{PCL}=$ Posttraumatic Stress Disorder Checklist will facilitate a conceptual understanding of the relationship between multiple treatment components related to different approaches of mindfulness and TF-CBT. This enables reporting on the role of not just one mechanism appropriate for the treatment in question, but also other putative mechanisms that make theoretical sense or for which previous evidence exists in the reduction of PTSS (Kazdin, 2007).

\section{Methods}

\section{Participants}

To reduce sample bias by collecting data from multiple institutions, convenience sampling was used; several university faculty members who were known to the author and had different affiliations throughout Japan were asked for assistance during recruitment. A total of 884 (381 men, 496 women, 7 unknown, $M_{\text {age }} 20.46 \pm 3.46$ years) Japanese undergraduate and graduate students were recruited from six universities in Japan. The survey was conducted during lecture periods between October 2019 and January 2020. All study participants provided written informed consent, and the study protocol was approved by the ethics review board of the corresponding institution (Approval number: 2019-23).

Trauma experience and PTSS were confirmed using the Trauma Experience Questionnaire (TEQ; Sato \& Sakano, 2001) and Post-Traumatic Stress Disorder Checklist (PCL; Ito, Horikoshi, \& Suzuki, 2015). As a result, 254 (106 men, 147 women, 1 unknown, $M_{\text {age }} 21.17 \pm 4.40$ years) students with PTSS who had experienced traumatic events more than a month prior (PCL score $>0$ ) were included in the analysis.
Participants reported traumatic experiences including interpersonal trauma such as bullying, violence, and sexual assault $(n=112 ; 44 \%)$; exposure to danger at work or school $(n=$ $56 ; 22 \%)$; family troubles such as abuse, domestic violence, or sudden death of a loved one $(n=41 ; 16 \%)$; injury or illness as a result of traffic accidents $(n=24 ; 9 \%)$; crime-related damage $(n=7 ; 3 \%)$; and natural disasters $(n=4 ; 2 \%)$. The average time since trauma experience was $30.50 \pm 37.07$ months.

\section{Measures}

\section{Trauma Experience}

The TEQ developed by Sato and Sakano (2001) was used in this study to assess the content and time of a trauma experience. The TEQ is often used in Japan to assess traumatic events in a community sample. The following instructions were given to the respondents: "Describe a traumatic event that continues to cause the same intense distress and discomfort today as it did at the time of the experience. For example, it could be an event that threatened your life, or your most stressful life event."

\section{Mindful Attention Awareness}

The Japanese version of the MAAS (Fujino, Kajimura, \& Nomura, 2015) developed by Brown and Ryan (2003) was used in this study to measure mindful attention awareness. The MAAS comprises a single-factor structure and a 15 item self-report questionnaire of dispositional mindfulness, in which respondents indicate their level of awareness and attention to present events and experiences on a 6-point 
Likert-type scale $(1=$ almost never to $6=$ almost always $)$. A mean score is calculated, where higher scores reflect greater self-reported attention and awareness, or "dispositional mindfulness." The MAAS exhibits good internal consistency and high test-retest reliability, and the convergent and discriminant validity has been evaluated (Brown \& Ryan, 2003).

\section{Cognitive Fusion}

The Japanese version of the Cognitive Fusion Questionnaire (CFQ; Shima, Kawai, Yanagihara, \& Kumano, 2016) developed by Gillanders et al. (2014) was used in this study to measure cognitive fusion. This assessed cognitive fusion and included questions relating to the extent to which people's thoughts were distressing and interfere with action. The CFQ comprises a single-factor structure and a 7-item self-report questionnaire, in which respondents indicate their responses on a 7-point Likert-type scale $(1=$ not at all to $7=$ always); higher scores indicate higher levels of cognitive fusion. The CFQ has good internal consistency and temporal stability, as well as good evidence of construct validity (Gillanders et al., 2014).

\section{Posttraumatic Negative Cognitions}

The Brief Version of the Posttraumatic Cognitions Inventory (PTCI-9; Wells et al., 2019) was used in this study to measure posttraumatic cognition by using the Japanese version of the PTCI-36 (Nagae et al., 2004) as a reference. The PTCI-9 comprises three subscales: negative cognitions about the self, negative cognitions about the world, and self-blame. The PTCI-9 comprises a 9-item self-report questionnaire and responses are rated using a 7-point Likert-type scale ( $1=$ totally disagree to $7=$ totally agree). Total PTCI-9 scores are calculated by summing the raw scores of the three subscales; higher scores indicate negative and problematic posttraumatic cognition. PTCI-9 total and subscale scores display good internal consistency and strong correlations with the PTCI-36 (Foa, Ehlers, Clark, Tolin, \& Orsillo, 1999). Previous findings demonstrated that the PTCI-9 is a reliable and valid measure of posttraumatic cognitions (Wells et al., 2019).

\section{Avoidance Coping}

A portion of the Ways of Coping Checklists-Revised (WCCL$\mathrm{R}$; Osawa \& Sakano, 2007) was used in this study to measure coping strategies when recalling traumatic experiences, specifically thought / emotional avoidance coping. The WCCL-R comprises five subscales: problem-focused coping, social support seeking, thought/emotional avoidance coping, self-blame coping, and positive-cognitive coping. The WCCL-R is a 32item self-report questionnaire and questions are rated using a 4-point Likert-type scale $(0=$ not at all to $3=$ always $)$; higher scores indicate frequent use of the particular method of coping. The WCCL-R has good reliability and validity (Osawa \& Sakano, 2007). In this study, we used seven items from the "thought/emotional avoidance coping" subscale, which are associated with PTSD symptoms (Osawa \& Sakano, 2007); higher scores indicate more use of avoidance coping.

\section{Posttraumatic Stress Symptoms}

The Japanese version of the PCL (Ito et al., 2015) developed by Weathers et al. (2013) was used in this study to measure PTSS. The questions are subdivided into four clusters: Cluster B (Intrusion), Cluster C (Avoidance), Cluster D (Mood and Cognition), and Cluster E (Arousal Activity). The PCL is a self-report measure that assesses the 20 PTSS outlined in the Diagnostic and Statistical Manual of Mental Disorders, 5th Edition (APA, 2013; Weathers et al., 2013) and questions are rated using a 5 -point Likert-type scale $(0=$ not at all to $4=$ always); higher scores indicate higher levels of PTSS. The PCL total score is calculated by summing the raw scores of the four subscales. A total score of 33 or higher is a recommendation for referral to a specialized agency for diagnosis and treatment of PTSD. The PCL exhibits good internal consistency, test-retest reliability, and convergent and discriminant validity (Weathers et al., 2013).

Due to ethical constraints, this study relied on the participants' understanding of a traumatic event. Hence, we cannot deny the possibility of some events not being traumatic. Therefore, from now on, when we describe the symptoms of the participants in this study, we will refer to them as PTSDlike symptoms, rather than PTSD symptoms (PTSS).

\section{Statistical Analysis}

All analyses were performed using SPSS and Amos ver. 25 (IBM Japan, Ltd.). Raw scores for each variable were analyzed, and Zero-order Pearson's correlations were computed between each measure. SEM was performed on the model using the maximum likelihood method as shown in Fig. 1, and the goodness of fit was calculated. Subsequently, a more plausible model was investigated at an exploratory level using the specification search method without significantly changing the theoretical framework.

\section{Results}

Table 1 displays descriptive statics for all measures. As shown in Table 1, the internal consistency of all measures in this study was generally high, and all measures were normally distributed. The average total PCL score $(M=24.68, S D=$ 16.73 ) was below the cut-off value of 33 points; however, 81 participants (32\%) scored 33 or more. 
Table 1 Psychometric properties of mindful attention awareness, cognitive fusion, posttraumatic cognitions, coping, and PTSDlike symptoms among Japanese with traumatic experiences

\begin{tabular}{lcccccc}
\hline Scale & Mean & SD & Range & $\alpha$ & Skewness & Kurtosis \\
\hline MASS & 59.15 & 10.72 & $15-90$ & .86 & .25 & .70 \\
CFQ & 27.60 & 9.94 & $7-49$ & .91 & .08 & -.60 \\
WCCL-R (Avoidance coping) & 10.37 & 5.28 & $0-21$ & .80 & .15 & -.88 \\
PTCI-9 (Total) & 31.76 & 11.92 & $9-63$ & .86 & .00 & -.50 \\
$\quad$ Negative cognitions about the self & 8.77 & 4.75 & $3-21$ & .80 & .47 & -.70 \\
$\quad$ Negative cognitions about the world & 10.93 & 5.01 & $3-21$ & .83 & .05 & -.93 \\
$\quad$ Self-blame & 12.05 & 5.12 & $3-21$ & .82 & -.35 & -.86 \\
PCL & 24.68 & 16.73 & $0-100$ & .93 & .00 & -.40 \\
\hline
\end{tabular}

Note: This shows descriptive statistics and internal consistency of each of the scales using the sample data from the trauma-experienced undergraduate and graduate students $(n=254)$ in this study. MAAS = Mindful Attention Awareness Scale, CFQ = Cognitive Fusion Questionnaire, WCCL-R = Ways of Coping Checklist-Revised, PTCI-9 = Brief Version of the Posttraumatic Cognitions Inventory, PCL $=$ Posttraumatic Stress Disorder Checklist
Table 2 displays the results of correlation analysis between each scale. Although there was a slightly higher negative correlation between mindful attention awareness and cognitive fusion $(r=-.58, p<.001)$, and a slightly higher positive correlation between cognitive fusion and posttraumatic negative cognitions $(r=.54, p<.001)$, other correlations among each variable were moderate, indicating that multicollinearity would be unlikely in the case of a multivariate analysis.

First, SEM was performed according to the theoretical model in Fig. 1 to examine the association of mindful attention awareness and cognitive fusion with PTSD-like symptoms mediated by posttraumatic negative cognitions and avoidance coping according to the theoretical model was good fit: $\chi^{2}$ $(8)=.11 .59, p=.17$; GFI $=.99$, $\mathrm{AGFI}=.95, \mathrm{CFI}=.99$, and RMSEA $=.04$. Therefore, the specification search was performed to determine whether these three paths were necessary, and the results showed a better-fitting model: $\chi^{2}(10)=.12 .3$,

Table 2 Associations between mindful attention awareness, cognitive fusion, posttraumatic cognitions, coping, and PTSD-like symptoms among Japanese with traumatic experiences

\begin{tabular}{lccccc}
\hline Scale & 1 & 2 & 3 & 4 & 5 \\
\hline 1. MAAS & - & & & & \\
2. CFQ & $-.58^{* * *}$ & - & & & \\
3. WCCL-R & $-.34^{* * *}$ & $.45^{* * *}$ & - & & \\
4. PTCI-9 & $-.44^{* * *}$ & $.54^{* * *}$ & $.46^{* * * *}$ & - \\
5. PCL & $-.49^{* * *}$ & $.61^{* * *}$ & $.48^{* * *}$ & $.68^{* * * *}$ & - \\
\hline
\end{tabular}

Note: This shows the results of the correlation analysis between each of the scales using the sample data from the trauma-experienced undergraduate and graduate students $(n=254)$ in this study. MAAS $=$ Mindful Attention Awareness Scale, CFQ $=$ Cognitive Fusion Questionnaire, WCCL-R = Ways of Coping Checklist-Revised (Avoidance coping), PTCI-9 = Brief Version of the Posttraumatic Cognitions Inventory, PCL $=$ Posttraumatic Stress Disorder Checklist

$* * * p<.001$ $p=.27, \mathrm{GFI}=.99, \mathrm{AGFI}=.96, \mathrm{CFI}=.99$, and $\mathrm{RMSEA}=.03$. The higher goodness of fit model was the model with two paths (pathway from mindful attention awareness to PTSDlike symptoms, pathway from avoidance coping to PTSD-like symptoms), which was removed from the model shown in Fig. 1. The final model of the completely standardized coefficients is shown in Fig. 2. Regarding the relationship between the scales for the model, mindful attention awareness had no direct association on PTSD-like symptoms or avoidance coping, contrary to the expectations, but had a strong negative association on cognitive fusion $(\beta=-.58,95 \% \mathrm{Cl}:[-.67$, $-.47], p<.001)$ and a weak negative association on posttraumatic negative cognitions $(\beta=-.21,95 \% \mathrm{Cl}$ : $[-.34,-.07]$, $p<.01)$. Furthermore, cognitive fusion had a moderate positive association on avoidance coping $(\beta=.39,95 \% \mathrm{Cl}$ : $[.26$, $.50], p<.001)$ and posttraumatic negative cognitions $(\beta=.36$, 95\% Cl: $[.21, .51], p<.001)$, and a weak positive association on PTSD-like symptoms directly $(\beta=.16,95 \% \mathrm{Cl}$ : $[.01, .30]$, $p<.05)$, as expected. Additionally, avoidance coping had no direct association on PTSD-like symptoms $(\beta=.02,95 \% \mathrm{Cl}$ : $[-.11, .15], p=.81)$, contrary to the expectation, but a moderate positive association on posttraumatic negative cognitions $(\beta=.33,95 \% \mathrm{Cl}:[.22, .44], p<.001)$. Posttraumatic negative cognitions had a strong positive association on PTSD-like symptoms $(\beta=.71,95 \% \mathrm{Cl}:[.58, .85], p<.001)$.

These results suggested no direct association of mindful attention awareness on PTSD-like symptoms, a small direct association of cognitive fusion on PTSD-like symptoms, and a large indirect association mediated by cognitive behavioral factors, such as avoidance coping and posttraumatic negative cognitions. A bias-corrected bootstrap analysis (1000 resamples) was conducted to reveal the significance of the indirect associations. The indirect associations of mindful attention awareness and cognitive fusion on PTSD-like symptoms were significant $(\beta=-.47,95 \% \mathrm{Cl}:[-.56,-.36], p<.001$; $\beta=.35,95 \% \mathrm{Cl}:[.23, .50], p<.001)$ and more intense than 


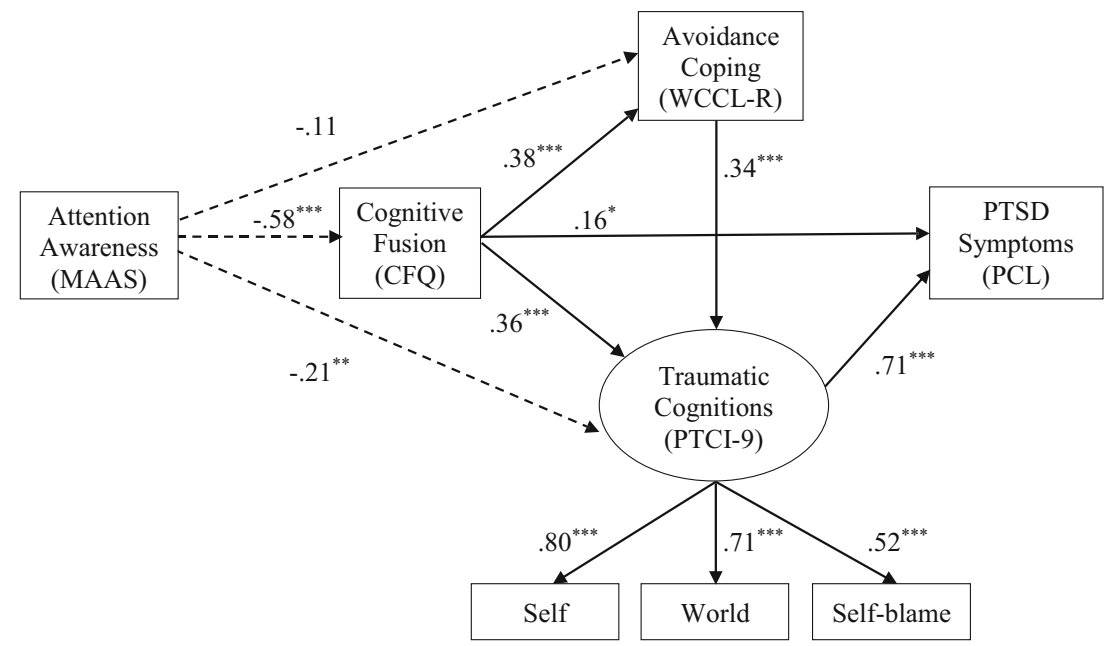

Fig. 2 The Result of a Structural Equation Modeling on the Association of Mindful Attention Awareness and Cognitive Fusion Associated with PTSD-like Symptoms via Avoidance Coping and Posttraumatic Cognitions among Japanese with Traumatic Experiences. Note. This shows the results of structural equation modelling on the association of mindfulness tendencies such as mindful attention and awareness (MAAS), and cognitive fusion (CFQ) with PTSS (PCL) mediated by posttraumatic negative cognition (PTCI-9) and avoidance coping (WCCL-R) using the sample data from the trauma-experienced

the direct associations of cognitive fusion on PTSD-like symptoms $(\beta=.16, p<.05)$. The indirect association of avoidance coping on PTSD-like symptoms was significant $(\beta=.24$, $95 \% \mathrm{Cl}:[.16, .33], p<.001)$, although there was no direct association.

\section{Discussion}

The purpose of this exploratory study was to examine the association of mindful attention awareness and cognitive fusion with PTSD-like symptoms mediated by posttraumatic negative cognition and avoidance coping. First, similar to previous studies (Boden et al., 2012; Nitzan-Assayag et al., 2015), correlation analysis results of this study indicated that mindful attention awareness and PTSD-like symptoms were highly negatively correlated and cognitive fusion and PTSDlike symptoms were highly positively correlated. Additionally, there was a relatively high positive correlation between cognitive-behavioral factors (posttraumatic cognition and avoidance coping) and PTSD-like symptoms, which is consistent with previous studies (Hembree \& Foa, 2000). These results are not surprising, given the large number of efficacy studies that demonstrate the effectiveness of mindfulness-based training (Boyd et al., 2018) and TF-CBT (Foa \& Rothbaum, 1998) for PTSS.

This was a cross-sectional study; therefore, conclusions about causality should be made with caution. However, the final model using SEM indicated no direct association of mindful attention awareness on PTSD-like symptoms, a small undergraduate and graduate students $(\mathrm{n}=254)$ in this study. The numbers represent a completely standardized beta coefficient. Dotted lines represent negative effect pathway and bold lines represent positive effect pathway. For clarity, error variables and co-variances are not depicted. MAAS = Mindful Attention Awareness Scale, $\mathrm{CFQ}=$ Cognitive Fusion Questionnaire, WCCL-R = Ways of Coping Checklist-Revised, PTCI$9=$ Brief Version of the Posttraumatic Cognitions Inventory, $\mathrm{PCL}=$ Posttraumatic Stress Disorder Checklist. ${ }^{*} p<.05,{ }^{* * *} p<.01,{ }^{* * * *} p<.001$

direct association of cognitive fusion on PTSD-like symptoms, and a large indirect association mediated by posttraumatic negative cognitions and avoidance coping. Notably, although correlation analysis confirmed a high correlation between mindful attention awareness and PTSD-like symptoms, the final model showed that the direct associated mindful attention awareness with PTSD-like symptoms disappeared, and it was indirectly associated with PTSD-like symptoms mediated by cognitive fusion, avoidance coping, and posttraumatic negative cognition. Additionally, the direct associations of cognitive fusion on PTSD-like symptoms were lessened, and indirectly affected PTSD-like symptoms by mediating avoidance coping and traumatic cognition. A bias-corrected bootstrap analysis revealed that the indirect associations of mindful attention awareness and cognitive fusion on PTSDlike symptoms, through mediation of avoidance coping and traumatic cognition, were relatively large, and even greater than the direct associations. These results support theoretic suggestions that improving the skills necessary to draw attention to the present moment and escape the cognitive fusion of reality and trauma related-thoughts will eventually promote coping methods other than avoidance (Lang et al., 2012) and cognitive modification (Sears \& Chard, 2016).

However, there are two major differences between the first model assumed from previous research and the final model. In the first model, although we predicted that mindful attention awareness would directly reduce PTSD-like symptoms, the results of SEM indicated that the direct associated mindful attention awareness with PTSD-like symptoms disappeared, and it was indirectly associated with PTSD-like symptoms 
mediated by cognitive fusion, avoidance coping, and posttraumatic negative cognition. This suggests that simply improving mindful attention awareness does not improve PTSD-like symptoms. Indeed, it suggested that encouraging patients with PTSD to pay attention to their thoughts, feelings, and bodily sensations could be an unpleasant experience and might even exacerbate PTSS, because their distress was often caused by them (Sears \& Chard, 2016). Furthermore, a small number of studies (Hagen, Lien, Hauff, \& Heir, 2016) that examined mindfulness using the Five Facet Mindfulness Questionnaire (Baer, Smith, Hopkins, Krietemeyer, \& Toney, 2006) showed that "observation" and "description" concepts similar to MAAS were not correlated or positively correlated with PTSS. Thus, previous studies have not produced consistent results regarding the association between mindful attention awareness and PTSS, and further investigation is needed. However, it may be important to note that mindful attention awareness affects not only one's own state of mind, but also whether attention to reality or cognitive defusion is subsequently encouraged.

Second, we predicted that avoidance coping directly worsens PTSD-like symptoms; the results of SEM indicated that the direct association of avoidance coping with PTSD-like symptoms disappeared, and it was indirectly associated with PTSD-like symptoms mediated by posttraumatic negative cognition. The results should be interpreted with caution because the avoidance coping scale used in this study (WCCL-R) was not specific to trauma survivors and assessed a narrow domain of thoughts and emotions, which may have influenced the results. However, emotional processing theories of PTSD have suggested that the activation of fear structures by confronting trauma-related stimuli is important because it adds new information that was not previously obtained through avoidance coping (Foa \& Kozak, 1986). Furthermore, recent clinical findings have pointed to the importance of cognitive factors as an effect mechanism for exposures that confronts trauma-related stimuli. For example, Craske, Treanor, Conway, Zbozinek, and Vervliet (2014) recommended an inhibitory learning approach in which exposure tasks were designed to accommodate "what do you need to learn" rather than simply stopping avoidance behaviors and "stay in the situation until fear declines" that would be predicated from a habituation-based model of exposure therapy. This might show that it is important to change cognition as a result, rather than simply stopping avoidance behavior and exposure to traumarelated stimuli.

Thus, although initial assumptions and the final model had some differences, we were able to indicate the processes by which mindfulness tendencies are mediated by cognitivebehavioral factors to influence PTSD-like symptoms. These results may support a recent systematic review (Kangaslampi \& Peltonen, 2019) that improvements in maladaptive traumarelated cognitions are a core factor in recovery from PTSS. The results also support the possibility that changes involve a reduction in avoidance behaviors due to exposure to traumatic stimuli and a reduction in susceptibility to threatening stimuli due to mindfulness. In other words, while some preliminary evidence suggests that reductions in PTSS achieved by mindfulness interventions are indeed associated with increases in dispositional mindfulness (Kangaslampi \& Peltonen, 2019), the effect may not be explained simply by increased mindfulness alone. Thus, mindfulness-based interventions may also be utilized as an adjunctive preparation for conventional TFCBT, such as exposure therapy (e.g., by increasing ability to tolerate experiencing emotions), an aid to cognitive therapies (e.g., by increasing engagement and developing cognitive skills), or possibly as an intervention to modulate emotional reactivity (King et al., 2013). Studies on the mechanism of certain symptoms, such as this study, are useful because they can separate general mechanisms from more specific ones. Identifying shared key mechanisms of change may explain how differing approaches may all lead to reduction in symptoms (Kazdin, 2007). Furthermore, testing the same model for the different mechanisms assumed will help clarify the role of each mechanism. Although it is significant that this study clarified the relationship between multiple psychological factors related to the different approaches of mindfulness and TFCBT and discussed their roles, it is necessary to investigate them using a more accurate research design in the future.

This study has some limitations. First, mindfulness comprises various elements (Baer et al., 2006); however, we focused on mindful attention and awareness and cognitive fusion as important factors, as they have consistently been associated with PTSS, compared to other factors (e.g., description). For example, a self-administered scale that measures mindfulness on a five-factor scale, such as the Five Facet Mindfulness Questionnaire (Baer et al., 2006), might be used to examine the relationship between cognitive-behavioral factors and the PTSS in detail. However, mindfulness assessment is without a gold standard (Park, Reilly-Spong, \& Gross, 2013), and there is a particular need to improve the accuracy of self-reported mindfulness. Assessments in this study were conducted using only self-report measures; thus, it would be necessary to assess mindfulness in a multifaceted and accurate manner, such as structured interviews, in the future. Second, the posterior test power of this study was acceptable $(1-\beta=.77)$, but somewhat low, and the study design was not a longitudinal survey, but a cross-sectional survey; therefore, strong conclusions cannot be drawn regarding the causal relationship of mindfulness and cognitive-behavioral factors. It has been pointed out that there are serious limitations and potential problems with mediation analysis in cross-sectional designs (e.g., Bullock, Green, \& Ha, 2010), especially regarding potential bias in findings and possibly unrealistic assumptions inherent in the methods. For example, cross-sectional analyses can imply the existence of a substantial indirect effect even when the true longitudinal indirect effect is zero 
(Maxwell, Cole, \& Mitchell, 2011). Thus, a variable that is found to be a strong mediator in a cross-sectional analysis may not be a mediator at all in a longitudinal analysis. The study may show that when the studied mediating factors are considered, mindful awareness is not directly associated with PTSS, but it is indirectly associated with PTSS through others. Therefore, future studies should increase the sample size and adopt a longitudinal or experimental design to clarify causality and generalizability (e.g., Bullock et al., 2010). Third, since this study did not target patients with a diagnosis of PTSD and used a limited community sample such as college students, the possibility of generalization needs to be examined in the future. In particular, because this study relied on participants' understanding of a traumatic event, we cannot completely rule out the possibility that the study may have included events that were not actually traumatic. Furthermore, the results of this study may not adequately reflect the specificity of trauma and PTSD, as the analyzed participants included those with mild PTSD-like symptoms. It is necessary to empirically examine whether the findings of this study can be applied to PTSD, although they may be applicable to some extent. PTSD is a graded dimensional model (Kliem et al., 2016), and 32\% of individuals with severe PTSS exceeded the PCL cut-off in this study. Furthermore, this study had a very small sample population in terms of age and socioeconomic background. Therefore, compared to other broad age groups, they were more likely to experience interpersonal problems such as bullying and violence, and traumatic experiences at work and school, and relatively less likely to experience trauma such as sexual assault, traffic accidents, and natural disasters. Additionally, findings showed that a lower educational history is associated with a higher risk of developing PTSD due to traumatic life experiences (Ozer, Best, Lipsey, \& Weiss, 2003). Therefore, a more rigorous screening for trauma and symptoms in a diverse and broad sample is needed to clarify the generalizability of the findings and their application to PTSD in the future. Fourth, mechanisms of change in mindfulness-based interventions may involve not only cognitive and behavioral factors, but also factors such as psychological flexibility, self-compassion (Meyer et al., 2019), and emotion regulation (Dick, Niles, Street, DiMartino, \& Mitchell, 2014). These factors and others will hopefully be included in future research to clarify the mechanisms of mindfulness change and how mindfulness can be used in the treatment of PTSD. Furthermore, in recent years, it has been recommended to develop treatments that are tailored to the needs of different patient groups regarding factors such as age, sex, culture, comorbidities, and type of trauma experience (Schnyder et al., 2015). For examples, previous attempts to culturally adapt the clinical intervention MBSR for diverse populations have met with mixed success; however, some data indicate interplay with treatments and that different cultures experience trauma differently, so these results show that it is essential to assess the acceptability of this type of intervention according to the cultural contexts in which it is provided (Hammerstein et al., 2019). Therefore, it is also necessary to consider how mindfulness-based treatment works according to the characteristics of the individual.

Acknowledgements We would like to thank Editage (www.editage.jp) for English. language editing.

Funding This work was supported by JSPS KAKENHI (grant Number JP19K03315).

\section{Data Availability}

The data that support the findings of this study are available from the corresponding author upon reasonable request.

\section{Declarations}

Conflict of Interest The authors declare that they have no conflict of interest.

Ethics Approval All study participants provided written informed consent, and the study protocol was approved by the ethics review board of the corresponding institution (Approval number: 2019-23).

Informed consent was obtained from all individual participants included in the study.

All individual participants agreed that the results of the analysis of the numeric data obtained from the questionnaire would be published.

Open Access This article is licensed under a Creative Commons Attribution 4.0 International License, which permits use, sharing, adaptation, distribution and reproduction in any medium or format, as long as you give appropriate credit to the original author(s) and the source, provide a link to the Creative Commons licence, and indicate if changes were made. The images or other third party material in this article are included in the article's Creative Commons licence, unless indicated otherwise in a credit line to the material. If material is not included in the article's Creative Commons licence and your intended use is not permitted by statutory regulation or exceeds the permitted use, you will need to obtain permission directly from the copyright holder. To view a copy of this licence, visit http://creativecommons.org/licenses/by/4.0/.

\section{References}

American Psychiatric Association. Diagnostic and statistical manual of mental disorders. (American Psychiatric Publishing, 2013).

Baer, R. A., Smith, G. T., Hopkins, J., Krietemeyer, J., \& Toney, L. (2006). Using self-report assessment methods to explore facets of mindfulness. Assessment, 13(1), 27-45. https://doi.org/10.1177/ 1073191105283504.

Becker, C. B., Darius, E., \& Schaumberg, K. (2007). An analog study of patient preferences for exposure versus alternative treatments for posttraumatic stress disorder. Behavior Research and Therapy, 45, 2861-2873. https://doi.org/10.1016/j.brat.2007.05.006.

Bernstein, A., Hadash, Y., Lichtash, Y., Tanay, G., Shepherd, K., \& Fresco, D. M. (2015). Decentering and related constructs: A critical review and metacognitive processes model. Perspectives on 
Psychological Science, 10(5), 599-617. https://doi.org/10.1177/ 1745691615594577.

Bishop, S. R., Lau, M., Shapiro, S., Carlson, L., Anderson, N. D., Carmody, J., Segal, Z. V., Abbey, S., Speca, M., Velting, D., \& Devins, G. (2004). Mindfulness: A proposed operational definition. Clinical Psychology: Science and Practice, 11(3), 230-241. https:// doi.org/10.1093/clipsy.bph077.

Boden, M. T., Bernstein, A., Walser, R. D., Bui, L., Alvarez, J., \& BonnMiller, M. O. (2012). Changes in facets of mindfulness and posttraumatic stress disorder treatment outcome. Psychiatry Research, 200(2-3), 609-613. https://doi.org/10.1016/j.psychres.2012.07. 011.

Boyd, J. E., Lanius, R. A., \& McKinnon, M. C. (2018). Mindfulnessbased treatments for posttraumatic stress disorder: A review of the treatment literature and neurobiological evidence. Journal of Psychiatry \& Neuroscience, 43(1), 7-25. https://doi.org/10.1503/ jpn. 170021

Bradley, R., Greene, J., Russ, E., Dutra, L., \& Westen, D. (2005). A multidimensional meta-analysis of psychotherapy for PTSD. The American Journal of Psychiatry, 162(2), 214-227. https://doi.org/ 10.1176/appi.ajp.162.2.214.

Broman-Fulks, J. J., Ruggiero, K. J., Green, B. A., Smith, D. W., Hanson, R. F., Kilpatrick, D. G., \& Saunders, B. E. (2009). The latent structure of PTSD among adolescents. Journal of Traumatic Stress, 22(2), 146-152. https://doi.org/10.1002/jts.20399.

Brown, K. W., \& Ryan, R. M. (2003). The benefits of being present: Mindfulness and its role in psychological well-being. Journal of Personality and Social Psychology, 84(4), 822-848. https://doi. org/10.1037/0022-3514.84.4.822.

Brown, K. W., Ryan, R. M., \& Creswell, J. D. (2007). Mindfulness: Theoretical foundations and evidence for its salutary effects. Psychological Inquiry, 18(4), 211-237. https://doi.org/10.1080/ 10478400701598298.

Bullock, J. G., Green, D. P., \& Ha, S. E. (2010). Yes, but what's the mechanism? (don't expect an easy answer). Journal of Personality and Social Psychology, 98(4), 550-558. https://doi.org/10.1037/ a0018933.

Craske, M. G., Treanor, M., Conway, C. C., Zbozinek, T., \& Vervliet, B. (2014). Maximizing exposure therapy: An inhibitory learning approach. Behaviour Research and Therapy, 58, 10-23. https://doi. org/10.1016/j.brat.2014.04.006.

Dick, A. M., Niles, B. L., Street, A. E., DiMartino, D. M., \& Mitchell, K. S. (2014). Examining mechanisms of change in a yoga intervention for women: The influence of mindfulness, psychological flexibility, and emotion regulation on PTSD symptoms. Journal of Clinical Psychology, 70(12), 1170-1182. https://doi.org/10.1002/jclp. 22104.

Ehlers, A., \& Clark, D. M. (2000). A cognitive model of persistent posttraumatic stress disorder. Behavior Research and Therapy, 38(4), 319-345. https://doi.org/10.1016/s0005-7967(99)00123-0.

Foa, E. B., Ehlers, A., Clark, D. M., Tolin, D. F., \& Orsillo, S. M. (1999). The posttraumatic cognitions inventory (PTCI): Development and validation. Psychological Assessment, 11(3), 303-314. https://doi. org/10.1037/1040-3590.11.3.303.

Foa, E. B., \& Kozak, M. J. (1986). Emotional processing of fear: Exposure to corrective information. Psychological Bulletin, 99(1), 20-35. https://doi.org/10.1037/0033-2909.99.1.20.

Foa EB., \& Rothbaum, B. O. (1998). Treating the trauma of rape: Cognitive-behavioral therapy for PTSD (treatment manuals for practitioners). Guilford Press.

Fujino, M., Kajimura, S., \& Nomura, M. (2015). Development and validation of the Japanese version of the mindful attention awareness scale using item response theory analysis. The Japanese Journal of Personality, 24(1), 61-76. https://doi.org/10.2132/personality.24. 61.
Gillanders, D. T., Bolderston, H., Bond, F. W., Dempster, M., Flaxman, P. E., Campbell, L., Kerr, S., Tansey, L., Noel, P., Ferenbach, C., Masley, S., Roach, L., Lloyd, J., May, L., Clarke, S., \& Remington, B. (2014). The development and initial validation of the cognitive fusion questionnaire. Behavior Therapy, 45(1), 83-101. https://doi. org/10.1016/j.beth.2013.09.001

Goetter, E. M., Bui, E., Ojserkis, R. A., Zakarian, R. J., Brendel, R. W., \& Simon, N. M. (2015). A systematic review of dropout from psychotherapy for posttraumatic stress disorder among Iraq and Afghanistan combat veterans. Journal of Trauma Stress, 28(5), 401-409. https://doi.org/10.1002/jts.22038.

Grossman, P., Nieman, L., Schmidt, S., \& Walach, H. (2004). Mindfulness-based stress reduction and health benefits: A metaanalysis. Journal of Psychosomatic Research, 57(1), 35-43. https://doi.org/10.1016/S0022-3999(03)00573-7.

Hagen, C., Lien, L., Hauff, E., \& Heir, T. (2016). Is mindfulness protective against PTSD? A neurocognitive study of 25 tsunami disaster survivors. Journal of Negative Results in Biomedicine, 15(1), 13. https://doi.org/10.1186/s12952-016-0056-x.

Hammerstein, C., Khazaal, Y., Dupuis, M., Aubin, H. J., Benyamina, A., Luquiens, A., \& Romo, L. (2019). Feasibility, acceptability and preliminary outcomes of a mindfulness-based relapse prevention program in a naturalistic setting among treatment-seeking patients with alcohol use disorder: A prospective observational study. $B M J$ Open, 9(5), e026839. https://doi.org/10.1136/bmjopen-2018026839.

Hayes, A. F., \& Rockwood, N. J. (2016). Regression-based statistical mediation and moderation analysis in clinical research: Observations, recommendations, and implementation. Behavior Research and Therapy, 98, 39-57. https://doi.org/10.1016/j.brat. 2016.11.001.

Hembree, E. A., \& Foa, E. B. (2000). Posttraumatic stress disorder: Psychological factors and psychosocial interventions. The Journal of Clinical Psychiatry, 61(7), 33-39.

Hembree, E. A., Foa, E. B., Dorfan, N. M., Street, G. P., Kowalski, J., \& $\mathrm{Tu}, \mathrm{X}$. (2003). Do patients drop out prematurely from exposure therapy for PTSD? Journal of Traumatic Stress, 16, 555-562. https://doi.org/10.1023/B:JOTS.0000004078.93012.7d.

Ito, M., Horikoshi, M., \& Suzuki, Y. (2015). The Japanese version of PCL-5. Cognitive processing therapy for PTSD: A comprehensive manual. Sogensha. (Resick, P. A., Monson, C. M., \& Chard, K. M.). The Guilford Press. (Original work published 2017).

Kabat-Zinn, J. (1990). Full catastrophe living: Using the wisdom of your body and mind to face stress, pain, and illness. New York: Dell Publishing.

Kabat-Zinn J. (1994). Wherever you go, there you are: Mindfulness meditation in everyday life. Hyperion.

Kangaslampi, S., \& Peltonen, K. (2019). Mechanisms of change in psychological interventions for posttraumatic stress symptoms: A systematic review with recommendations. Current Psychology. https:// doi.org/10.1007/s12144-019-00478-5.

Kazdin, A. E. (2007). Mediators and mechanisms of change in psychotherapy research. Annual Review of Clinical Psychology, 3, 1-27. https://doi.org/10.1146/annurev.clinpsy.3.022806.091432.

Kilpatrick, D. G., Resnick, H. S., Milanak, M. E., Miller, M. W., Keyes, K. M., \& Friedman, M. J. (2013). National estimates of exposure to traumatic events and PTSD prevalence using DSM-IV and DSM-5 criteria. Journal of Trauma Stress, 26(5), 537-547. https://doi.org/ $10.1002 /$ jts. 21848 .

King, A. P., Erickson, T. M., Giardino, N. D., Favorite, T., Rauch, S. A., Robinson, E., et al. (2013). A pilot study of group mindfulnessbased cognitive therapy (MBCT) for combat veterans with posttraumatic stress disorder (PTSD). Depression and Anxiety, 30(7), 638645. https://doi.org/10.1002/da.22104.

Kliem, S., Kröger, C., Foran, H. M., Mößle, T., Glaesmer, H., Zenger, M., \& Brähler, E. (2016). Dimensional latent structure of PTSD- 
symptoms reporting: Is it adding by subtracting? Psychological Assessment, 28(12), 1663-1673. https://doi.org/10.1037/ pas0000287.

Lang, A. J., Strauss, J. L., Bomyea, J., Bormann, J. E., Hickman, S. D., \& Good, R. C. (2012). The theoretical and empirical basis for meditation as an intervention for PTSD. Behavior Modification, 36(6), 759-786. https://doi.org/10.1177/0145445512441200.

Marshall, R. D., Olfson, M., Hellman, F., Blanco, C., Guardino, M., \& Struening, E. L. (2001). Comorbidity, impairment, and Suicidality in subthreshold PTSD. American Journal of Psychiatry, 158(9), 1467-1473. https://doi.org/10.1176/appi.ajp.158.9.1467.

Maxwell, S. E., Cole, D. A., \& Mitchell, M. A. (2011). Bias in crosssectional analyses of longitudinal mediation: Partial and complete mediation under an autoregressive model. Multivariate Behavioral Research, 46(5), 816-841. https://doi.org/10.1080/00273171.2011. 606716.

Meyer, E. C., Szabo, Y. Z., Frankfurt, S. B., Kimbrel, N. A., DeBeer, B. B., \& Morissette, S. B. (2019). Predictors of recovery from postdeployment posttraumatic stress disorder symptoms in war veterans: The contributions of psychological flexibility, mindfulness, and selfcompassion. Behaviour Research and Therapy, 114, 7-14. https:// doi.org/10.1016/j.brat.2019.01.002.

Nagae, N., Masuda, T., Yamada, A., Kanetski, M., Nedate, K., \& Kim, Y. (2004). Development of a Japanese version of the posttraumatic cognition inventory (JPTCI): Prevalence of negative life events Among University students. Japanese Association of Behavioral and Cognitive Therapies, 30(2), 113-124. https://doi.org/10. 24468/jjbt.30.2_113.

Nitzan-Assayag, Y., Aderka, I. M., \& Bernstein, A. (2015). Dispositional mindfulness in trauma recovery: Prospective relations and mediating mechanisms. Journal of Anxiety Disorders, 36, 25-32. https:// doi.org/10.1016/j.janxdis.2015.07.008.

Osawa, K., \& Sakano, Y. (2007). Coping strategies towards traumatic memory recall and their effect on traumatic stress responses: A multiple regression study. The Japanese Journal of Stress Sciences, 21(4), 223-232.

Ozer, E. J., Best, S. R., Lipsey, T. L., \& Weiss, D. S. (2003). Predictors of Posttraumatic Stress Disorder and Symptoms in Adults: A MetaAnalysis. Psychological Bulletin, 129(1), 52-73. https://doi.org/10. 1037/0033-2909.129.1.52.

Park, T., Reilly-Spong, M., \& Gross, C. R. (2013). Mindfulness: A systematic review of instruments to measure an emergent patientreported outcome (PRO). Quality of Life Research, 22(10), 26392659. https://doi.org/10.1007/s11136-013-0395-8.

Reijnen, A., Rademaker, A. R., Vermetten, E., \& Geuze, E. (2015). Prevalence of mental health symptoms in Dutch military personnel returning from deployment to Afghanistan: A 2-year longitudinal analysis. European Psychiatry, 30(2), 341-346. https://doi.org/10. 1016/j.eurpsy.2014.05.003.

Sato, K., \& Sakano, Y. (2001). The relationship among disclosure of traumatic experiences, changes in distress on the traumatic experiences and physical symptoms. The Japanese Journal of Counseling Science, 34, 1-8.

Schnyder, U., Ehlers, A., Elbert, T., Foa, E. B., Gersons, B. P. R., Resick, P., Shapiro, F., et al. (2015). Psychotherapies for PTSD: What do they have in common? European Journal of Psychotraumatology, 6, 28186. https://doi.org/10.3402/ejpt.v6.28186.

Sears, R.W. \& Chard, K.M. (2016). Mindfulness-based cognitive therapy for posttraumatic stress disorder. John Wiley \& Sons.

Shapiro, S. L., Carlson, L. E., Astin, J. A., \& Freedman, B. (2006). Mechanisms of mindfulness. Journal of Clinical Psychology, 62, 73-86. https://doi.org/10.1002/jclp.20237.

Shima, T., Kawai, T., Yanagihara, M., \& Kumano, H. (2016). Validation of the Japanese version of the cognitive fusion questionnaire: The 13 -item content revised version and the 7-item version. The Japanese Journal of Behavior Therapy, 42(1), 73-83. https://doi. org/10.24468/jjbt.42.1 73 .

Steenkamp, M. M., Litz, B. T., Hoge, C. W., \& Marmar, C. R. (2015). Psychotherapy for military-related PTSD: A review of randomized clinical trials. Journal of the American Medical Association, 314(5), 489-500. https://doi.org/10.1001/jama.2015.8370.

Thompson, R. W., Arnkoff, D. B., \& Glass, C. R. (2011). Conceptualizing mindfulness and acceptance as components of psychological resilience to trauma. Trauma, Violence \& Abuse, 12(4), 220-235. https://doi.org/10.1177/1524838011416375.

Wells, S. Y., Morland, L. A., Torres, E. M., Kloezeman, K., Machitosh, M. A., \& Aarons, G. A. (2019). The development of a brief version of the posttraumatic cognitions inventory (PTCI-9). Assessment, 26(2), 198-208. https://doi.org/10.1177/1073191116685401.

Weathers, F. W., Lits, B. T., Kean, T. M., Palmieri, P. A., Marx, B. P., \& Schnurr, P. P. (2013). The PTSD checklist for DSM-5 (PCL-5), Scale available from National Center for PTSD, www.ptsd.va.gov (2013).

Westen, D. (1999). Psychology: Mind, brain, and culture (2nd ed.). New York: Wiley.

Xue, C., Ge, Y., Tang, B., Liu, Y., Kang, P., Wang, M., \& Zhang, L. (2015). A meta-analysis of risk factors for combat-related PTSD among military personnel and veterans. PLoS One, 10(3), e0120270. https://doi.org/10.1371/journal.pone.0120270.

Zalta, A. K., Gillihan, S. J., Fisher, A. J., Mintz, J., McLean, C. P., Yehuda, R., \& Foa, E. B. (2014). Change in negative cognitions associated with PTSD predicts symptom reduction in prolonged exposure. Journal of Consulting and Clinical Psychology, 82(1), 171-175. https://doi.org/10.1037/a0034735.

Zayfert, C., Deviva, J. C., Becker, C. B., Pike, J. L., Gillock, K. L., \& Hayes, S. A. (2005). Exposure utilization and completion of cognitive behavioral therapy for PTSD in a "real world" clinical practice. Journal of Trauma Stress, 18(6), 637-645. https://doi.org/10.1002/ jts.20072.

Publisher's Note Springer Nature remains neutral with regard to jurisdictional claims in published maps and institutional affiliations. 